\title{
The Anisotropic Heisenberg Model in the Long-Range Interaction Limit
}

\author{
Paul A. Pearce and Colin J. Thompson \\ Mathematics Department, University of Melbourne, Parkville, Victoria, Australia
}

Received September 24, 1974

\begin{abstract}
We consider the general spin quantum mechanical anisotropic Heisenberg model on a $v$-dimensional lattice with potential $\gamma^{v} \varrho(\gamma|\boldsymbol{r}|)$ of Kac type. In the limit $\gamma \rightarrow 0+$ (after the thermodynamic limit) it is shown that the free energy is equivalent to the corresponding Curie-Weiss or mean-field expression.
\end{abstract}

\section{Introduction}

In 1966 Lebowitz and Penrose [1] rigorously established the van der WaalsMaxwell theory for a classical system of particles in $v$-dimensions interacting with pair potential $v(\boldsymbol{r})$ of Kac type

$$
v(\boldsymbol{r})=q(\boldsymbol{r})+\gamma^{v} \varrho(\gamma \boldsymbol{r})
$$

in the limit $\gamma \rightarrow 0+$ (after the thermodynamic limit), for short range repulsive (hard core) potentials $q(\boldsymbol{r})$ and long-range attractive potentials $\gamma^{v} \varrho(\gamma \boldsymbol{r})$ satisfying conditions stated in [1] (and in the following section). In the same year Lieb [2] obtained the same result for quantum systems of particles.

Ising spin systems (or equivalently lattice gases) can be treated in the same way [3], with spin $-\frac{1}{2}$ Curie-Weiss theory of magnetism resulting in the limit $\gamma \rightarrow 0+$.

Different methods were used recently [4] to investigate the $\gamma \rightarrow 0+$ limit of $n$-vector classical spin systems interacting with a Kac potential. The resulting thermodynamics in this case was shown to be identical to that of an equivalent neighbor or Curie-Weiss model in which all spins interact equally with one another [5], with coupling constant inversely proportional to the number of spins. As a special case, with $n=1$ (corresponding to Ising systems) one recovers the spin $-\frac{1}{2}$ Curie-Weiss theory.

Our purpose here is to extend the methods of ref. [4] to investigate the $\gamma \rightarrow 0+$ limit of the general spin anisotropic Heisenberg model.

The main results are stated and discussed in the following section. Sections Three and Four are devoted to the proof of the theorem stated in Section Two.

\section{Statement and Discussion of Results}

Consider a system of $N$ spins occupying the vertices of a $v$-dimensional lattice with Hamiltonian

$$
\mathscr{H}=-s^{-2} \sum_{1 \leqq i<j \leqq N} \varrho_{i j} S_{i} \cdot \boldsymbol{J} \cdot \boldsymbol{S}_{j}-s^{-1} \boldsymbol{H} \cdot \sum_{i=1}^{N} \boldsymbol{S}_{i}
$$


where

$$
S_{i}=\left(S_{i}^{x}, S_{i}^{y}, S_{i}^{z}\right)
$$

is the usual angular momentum operator for the $i^{\text {th }}$ spin, with total angular momentum $s$, i.e.

$$
S_{i} \cdot S_{i}=\left(S_{i}^{x}\right)^{2}+\left(S_{i}^{y}\right)^{2}+\left(S_{i}^{z}\right)^{2}=s(s+1)
$$

and $\boldsymbol{H}$ is the external magnetic field. The factors $s^{-2}$ and $s^{-1}$ multiplying the first and second terms in (2.1) are chosen so that the classical Heisenberg model results in the limit $s \rightarrow \infty[6,7]$. For simplicity we have introduced dyadic notation in (2.1) so that

$$
S_{i} \cdot \boldsymbol{J} \cdot \boldsymbol{S}_{j}=\sum_{\alpha, \beta=x, y, z} S_{i}^{\alpha} J^{\alpha \beta} S_{j}^{\beta} .
$$

(In the sequel $\boldsymbol{J} \cdot \boldsymbol{x}$ denotes a vector with components

$$
(\boldsymbol{J} \cdot \boldsymbol{x})^{\alpha}=\sum_{\beta=x, y, z} J^{\alpha \beta} x^{\beta}
$$

while $\boldsymbol{H} \cdot \boldsymbol{S}_{i}$ in (2.1) denotes ordinary scalar product.)

Since $i=j$ terms in (2.1) are excluded, the anisotropy tensor $J^{\alpha \beta}$ can be taken to be symmetric so that a representation for the spin operators can always be chosen so that only diagonal terms $\alpha=\beta$ appear in (2.4). Although this is the conventional way of expressing (2.1) we will adopt the form (2.4) in the following section and revert to a diagonal representation in section four.

Henceforth we will assume that the tensor $\boldsymbol{J}$ is symmetric and positive definite and that the interaction potentional $\varrho_{i j}$ is ferromagnetic and of Kac type,

$$
\varrho_{i j}=\gamma^{v} \varrho\left(\gamma\left|\boldsymbol{r}_{i}-\boldsymbol{r}_{j}\right|\right) \geqq 0
$$

where $\boldsymbol{r}_{i}$ is the position vector of the $i^{\text {th }}$ lattice site. In addition we will assume that the sum

$$
g(0, \gamma)=\gamma^{\nu} \sum_{\boldsymbol{l}} \varrho(\gamma|\boldsymbol{l}|)
$$

over the infinite lattice exists for all $\gamma>0$ and that

$$
g(0)=\lim _{\gamma \rightarrow 0+} g(0, \gamma)=\int \varrho(|\boldsymbol{r}|) d \boldsymbol{r}
$$

exists (as a Riemann integral).

The partition function is defined by

$$
Z_{N}(\gamma)=\operatorname{Tr} \exp (-\beta \mathscr{H})
$$

where $\beta=(k T)^{-1}$, and the limiting free energy per spin $\psi(\gamma)$ by

$$
-\beta \psi(\gamma)=\lim _{N \rightarrow \infty} N^{-1} \log Z_{N}(\gamma)
$$

Conditions on $\varrho(x)$ for the limit (2.10) to exist [8] will be assumed throughout.

We will be particularly concerned with the free energy $\psi_{0}$ in the long-range limit defined by

$$
\psi_{0}=\lim _{\gamma \rightarrow 0+} \psi(\gamma) .
$$

Our main result is the following. 
Theorem. For a system of spins with Hamiltonian (2.1) and potential $\varrho_{i j}$ satisfying conditions (2.6)-(2.8),

$$
\psi_{0}=\min _{\boldsymbol{x}}\left\{\frac{1}{2} g(0) \boldsymbol{x} \cdot \boldsymbol{J} \cdot \boldsymbol{x}-\beta^{-1} \log C_{s}(\beta\|g(0) \boldsymbol{J} \cdot \boldsymbol{x}+\boldsymbol{H}\|)\right\}
$$

where

$$
C_{s}(s \alpha)=\sinh \left(s+\frac{1}{2}\right) \alpha / \sinh \alpha / 2 \text {. }
$$

The minimum in (2.12) is attained for $\boldsymbol{x}$ a solution of

$$
\boldsymbol{x}=B_{s}(\beta\|g(0) \boldsymbol{J} \cdot \boldsymbol{x}+\boldsymbol{H}\|) \frac{g(0) \boldsymbol{J} \cdot \boldsymbol{x}+\boldsymbol{H}}{\|g(0) \boldsymbol{J} \cdot \boldsymbol{x}+\boldsymbol{H}\|}
$$

where $B_{s}$ is the Brillouin function of order $s$ defined by

$$
B_{s}(s \alpha)=\frac{2 s+1}{2 s} \operatorname{coth}\left(s+\frac{1}{2}\right) \alpha-\frac{1}{2 s} \operatorname{coth} \frac{\alpha}{2} \text {. }
$$

In the limit $\gamma \rightarrow 0+$ (after the thermodynamic limit) $s \boldsymbol{x}$ is in fact the total magnetic dipole moment (or magnetization) per spin.

Expressions (2.12) and (2.14) agree precisely with the spin-s Curie-Weiss theory $[9,10]$ and may be obtained from the corresponding equivalent neighbor model (or Curie-Weiss model) with $\varrho_{i j}$ in (2.1) replaced by $g(0) / N$. Kittel and Shore [11] and Niemeyer [12] in particular, examined the isotropic $\left(J^{\alpha \beta}=J \delta_{\alpha, \beta}\right)$ spin $-\frac{1}{2}$ equivalent neighbor model, observing that in the thermodynamic limit, the free energy is identical with that of the corresponding Ising-model. For general spin, $\boldsymbol{x}$ from (2.14) is parallel to $\boldsymbol{H}$ when the interaction is isotropic. In this case (2.12) becomes

$$
\psi_{0}=\frac{1}{2} g(0) J\|\boldsymbol{x}\|^{2}-\beta^{-1} \log C_{s}[\beta(g(0) J\|\boldsymbol{x}\|+\|\boldsymbol{H}\|)]
$$

where $\|\boldsymbol{x}\|$ is the non-negative solution of

$$
\|\boldsymbol{x}\|=B_{s}[\beta(g(0) J\|\boldsymbol{x}\|+\|\boldsymbol{H}\|)] .
$$

Expression (2.16) can also be obtained from an equivalent neighbor spin-s Ising system.

In the anisotropic case the magnetization from (2.14) is not necessarily parallel to $\boldsymbol{H}$. We will not examine the minimizing solutions of (2.14) (i.e. the magnetization) here. The interested reader is referred to Vertogen and De Vries [13] where special anisotropic spin $-\frac{1}{2}$ cases of (2.14) are examined in detail.

To prove the theorem we adopt the format of [4] and obtain upper and lower bounds on the free energy $\psi(\gamma)$ eq. (2.10) that coalesce to give the stated result (2.12) in the limit $\gamma \rightarrow 0+$. Our upper bound in the following section is obtained by an application of Bogoliubov's variational principle. The lower bound in Section Four is obtained via the Trotter formula [14] and a functional integral representation of the Trotter approximation to the partition function.

After this work was completed we obtained preprints of closely similar work by Tindemans and Capel $[15,16]$ on quantum mechanical systems with separable interactions. Although their techniques are similar to ours the precise form of the Heisenberg model Hamiltonian allows us to obtain precise bounds without resorting to saddle point methods used by Tindemans and Capel. 


\section{Upper Bound on the Free Energy}

For simplicity we impose periodic boundary conditions on the potential $\varrho_{i j}$ so that

$$
\sum_{j=1}^{N} \varrho_{i j}=g_{N}(0, \gamma)
$$

for all $i=1,2, \ldots, N$, and from (2.6) and (2.7)

$$
\lim _{N \rightarrow \infty} g_{N}(0, \gamma)=g(0, \gamma)
$$

We write the Hamiltonian $(2.1)$ as $\left(\varrho_{i i}=0\right)$

$$
\begin{aligned}
\mathscr{H}= & -\frac{1}{2} \sum_{i, j=1}^{N} \varrho_{i j}\left(\boldsymbol{\sigma}_{i}-\boldsymbol{x}\right) \cdot \boldsymbol{J} \cdot\left(\boldsymbol{\sigma}_{j}-\boldsymbol{x}\right)-\frac{1}{2} \boldsymbol{x} \cdot \sum_{i, j=1}^{N} \varrho_{i j} \boldsymbol{J} \cdot\left(\boldsymbol{\sigma}_{i}+\boldsymbol{\sigma}_{j}\right) \\
& +\frac{1}{2} \boldsymbol{x} \cdot \boldsymbol{J} \cdot \boldsymbol{x} \sum_{i, j=1}^{N} \varrho_{i j}-\boldsymbol{H} \cdot \sum_{i=1}^{N} \boldsymbol{\sigma}_{i}
\end{aligned}
$$

where $\boldsymbol{\sigma}_{i}=s^{-1} \boldsymbol{S}_{i}$ and for the moment $\boldsymbol{x}$ is arbitrary. Next we decompose $\mathscr{H}$ as

where from (3.1)

$$
\mathscr{H}=\mathscr{H}_{0}+\mathscr{H}_{1}
$$

$$
\mathscr{H}_{0}=\frac{1}{2} N g_{N}(0, \gamma) \boldsymbol{x} \cdot \boldsymbol{J} \cdot \boldsymbol{x}-\left(g_{N}(0, \gamma) \boldsymbol{J} \cdot \boldsymbol{x}+\boldsymbol{H}\right) \cdot \sum_{i=1}^{N} \boldsymbol{\sigma}_{i}
$$

and

$$
\mathscr{H}_{1}=-\frac{1}{2} \sum_{i, j=1}^{N} \varrho_{i j}\left(\boldsymbol{\sigma}_{i}-\boldsymbol{x}\right) \cdot \boldsymbol{J} \cdot\left(\boldsymbol{\sigma}_{j}-\boldsymbol{x}\right)
$$

We now appeal to the convexity inequality $[17,18]$ :

Bogoliubov's Variational Principle. If $\mathscr{H}_{0}$ and $\mathscr{H}_{1}$ are linear Hermitian operators acting on a separable Hilbert space then

where

$$
\operatorname{Tr} \exp \left[-\beta\left(\mathscr{H}_{0}+\mathscr{H}_{1}\right)\right] \geqq \exp \left(-\beta\left\langle\mathscr{H}_{1}\right\rangle_{0}\right) \operatorname{Tr} \exp \left(-\beta \mathscr{H}_{0}\right)
$$

$$
\langle\ldots\rangle_{0}=\operatorname{Tr}\left[\ldots \exp \left(-\beta \mathscr{H}_{0}\right)\right] / \operatorname{Tr} \exp \left(-\beta \mathscr{H}_{0}\right) .
$$

Since $\mathscr{H}_{0}$ and $\mathscr{H}_{1}$ are linear and Hermitian and act on the space $\bigotimes_{i=1}^{N} \mathbb{C}^{2 s+1}$, application of (3.7) to (2.9) gives

where

$$
\log Z_{N}(\gamma) \geqq \log Z_{N}^{0}(\gamma)+\left\langle\frac{\beta}{2} \sum_{i, j=1}^{N} \varrho_{i j}\left(\boldsymbol{\sigma}_{i}-\boldsymbol{x}\right) \cdot \boldsymbol{J} \cdot\left(\boldsymbol{\sigma}_{j}-\boldsymbol{x}\right)\right\rangle_{0}
$$

$$
\begin{aligned}
Z_{N}^{0}(\gamma)= & \operatorname{Tr} \exp \left(-\beta \mathscr{H}_{0}\right) \\
= & \exp \left(-\frac{\beta}{2} N g_{N}(0, \gamma) \boldsymbol{x} \cdot \boldsymbol{J} \cdot \boldsymbol{x}\right) \\
& \left\{\operatorname{Tr} \exp \left[\frac{\beta}{S}\left(g_{N}(0, \gamma) \boldsymbol{J} \cdot \boldsymbol{x}+\boldsymbol{H}\right) \cdot \boldsymbol{S}\right]\right\}^{N}
\end{aligned}
$$

and $S$ is a (single) spin-s vector operator. 
Using the facts that

$$
\left\langle\boldsymbol{\sigma}_{i} \cdot \boldsymbol{\sigma}_{j}\right\rangle_{0}=\left\langle\boldsymbol{\sigma}_{i}\right\rangle_{0} \cdot\left\langle\boldsymbol{\sigma}_{j}\right\rangle_{0} \text { for } i \neq j
$$

and $\varrho_{i i}=0,(3.9)$ gives

$$
\log Z_{N}(\gamma) \geqq \log Z_{N}^{0}(\gamma)+\frac{\beta}{2} \sum_{i, j=1}^{N} \varrho_{i j}\left(\left\langle\boldsymbol{\sigma}_{i}\right\rangle_{0}-\boldsymbol{x}\right) \cdot \boldsymbol{J} \cdot\left(\left\langle\boldsymbol{\sigma}_{j}\right\rangle_{0}-\boldsymbol{x}\right) .
$$

To obtain the desired bound we choose, since $\left\langle\boldsymbol{\sigma}_{i}\right\rangle_{0}=\langle\boldsymbol{\sigma}\rangle_{0}$ is a function of $\boldsymbol{x}$ independent of $i, \boldsymbol{x}$ a solution of

so that from (3.11)

$$
\boldsymbol{x}=\langle\boldsymbol{\sigma}\rangle_{0}
$$

$$
\log Z_{N}(\gamma) \geqq \log Z_{N}^{0}(\gamma)
$$

To evaluate $Z_{N}^{0}(\gamma)$ and $\langle\boldsymbol{\sigma}\rangle_{0}$ we need the results

$$
\begin{aligned}
\operatorname{Tr} \exp (\boldsymbol{\alpha} \cdot \boldsymbol{S}) & =\sum_{m=-s}^{s} \exp (\|\boldsymbol{\alpha}\| m) \\
& =C_{s}(s\|\boldsymbol{\alpha}\|)
\end{aligned}
$$

where $C_{s}(s \alpha)$ is defined by (2.13), and

$$
\operatorname{Tr}[S \exp (\boldsymbol{\alpha} \cdot S)]=s C_{s}^{\prime}(s\|\boldsymbol{\alpha}\|) \hat{\boldsymbol{\alpha}}
$$

which follows from (3.14) on differentiating with respect to $\boldsymbol{\alpha}$.

From the definitions of $Z_{N}^{0}(\gamma),(3.10)$ and $\langle\boldsymbol{\sigma}\rangle_{0}$, (3.12) we obtain from (3.14) and (3.15) respectively,

and

$$
Z_{N}^{0}(\gamma)=\exp \left(-\frac{1}{2} \beta N g_{N}(0, \gamma) \boldsymbol{x} \cdot \boldsymbol{J} \cdot \boldsymbol{x}\right)\left\{C_{s}\left(\beta\left\|g_{N}(0, \gamma) \boldsymbol{J} \cdot \boldsymbol{x}+\boldsymbol{H}\right\|\right)\right\}^{N}
$$

$$
\begin{aligned}
\boldsymbol{x} & =\frac{C_{s}^{\prime}\left(\beta\left\|g_{N}(0, \gamma) \boldsymbol{J} \cdot \boldsymbol{x}+\boldsymbol{H}\right\|\right)}{C_{s}\left(\beta\left\|g_{N}(0, \gamma) \boldsymbol{J} \cdot \boldsymbol{x}+\boldsymbol{H}\right\|\right)} \cdot \frac{g_{N}(0, \gamma) \boldsymbol{J} \cdot \boldsymbol{x}+\boldsymbol{H}}{\left\|g_{N}(0, \gamma) \boldsymbol{J} \cdot \boldsymbol{x}+\boldsymbol{H}\right\|} \\
& =B_{s}\left(\beta\left\|g_{N}(0, \gamma) \boldsymbol{J} \cdot \boldsymbol{x}+\boldsymbol{H}\right\|\right) \cdot \frac{g_{N}(0, \gamma) \boldsymbol{J} \cdot \boldsymbol{x}+\boldsymbol{H}}{\left\|g_{N}(0, \gamma) \boldsymbol{J} \cdot \boldsymbol{x}+\boldsymbol{H}\right\|}
\end{aligned}
$$

where $B_{s}(s \alpha)$ is defined by $(2.15)$.

It then follows from (3.13), (3.16) and (3.17) that

$$
\begin{aligned}
\psi_{0} & =\lim _{\gamma \rightarrow 0+} \lim _{N \rightarrow \infty}\left[-(\beta N)^{-1} \log Z_{n}(\gamma)\right] \\
& \leqq \frac{1}{2} g(0) \boldsymbol{x} \cdot \boldsymbol{J} \cdot \boldsymbol{x}-\beta^{-1} \log C_{s}(\beta\|g(0) \boldsymbol{J} \cdot \boldsymbol{x}+\boldsymbol{H}\|)
\end{aligned}
$$

where $g(0)$ is defined by (2.8), and $\boldsymbol{x}$ is a solution of the stationary condition (2.14).

In particular we conclude that

$$
\psi_{0} \leqq \min _{\boldsymbol{x}}\left\{\frac{1}{2} g(0) \boldsymbol{x} \cdot \boldsymbol{J} \cdot \boldsymbol{x}-\beta^{-1} \log C_{s}(\beta\|g(0) \boldsymbol{J} \cdot \boldsymbol{x}+\boldsymbol{H}\|)\right\}
$$




\section{Lower Bound for the Free Energy}

We begin by observing that the partition function (2.9) satisfies

$$
\begin{aligned}
Z_{N}(\gamma) & \leqq \operatorname{Tr} \exp \left[\frac{\beta}{2} \sum_{i, j=1}^{N} \varrho_{i j} \boldsymbol{\sigma}_{i} \cdot \boldsymbol{J} \cdot \boldsymbol{\sigma}_{j}+\beta \boldsymbol{H} \cdot \sum_{i=1}^{N} \boldsymbol{\sigma}_{i}\right] \\
& =\operatorname{Tr} \exp \left[\mathscr{H}\left(\sigma^{x}\right)+\mathscr{H}\left(\sigma^{y}\right)+\mathscr{H}\left(\sigma^{z}\right)\right]
\end{aligned}
$$

where in (4.1) a diagonal term $(i=j)$ has been added in the exponent, with $\varrho(0)$ chosen (sufficiently large) to make the matrix $\varrho_{i j}$ positive definite. The inequality results form Peierls' theorem since the operators $\boldsymbol{\sigma}_{i} \cdot \boldsymbol{J} \cdot \boldsymbol{\sigma}_{i}$ are positive definite. The separation (4.2) of the dependence of the Hamiltonian on spin components is then possible in principle co-ordinates where $\boldsymbol{J}=\operatorname{diag}\left(J^{x}, J^{y}, J^{z}\right)$ and for $\alpha=x, y, z$

$$
\mathscr{H}\left(\sigma^{\alpha}\right)=\frac{1}{2} \beta J^{\alpha} \sum_{i, j=1}^{N} \varrho_{i j} \sigma_{i}^{\alpha} \sigma_{j}^{\alpha}+\beta H^{\alpha} \sum_{i=1}^{N} \sigma_{i}^{\alpha} .
$$

Trotter's formula [14] asserts that the right hand side of (4.2) equals

$$
\lim _{n \rightarrow \infty} Z_{N, n}(\gamma)
$$

where

$$
Z_{N, n}(\gamma)=r e \operatorname{Tr}\left[e^{\mathscr{H}\left(\sigma^{x}\right) / n} e^{\mathscr{H}\left(\sigma^{y}\right) / n} \cdot e^{\mathscr{H}\left(\sigma^{z}\right) / n}\right]^{n} .
$$

We are now in a position to use the identity

$$
\begin{aligned}
\exp \left(\frac{\beta}{2} \sum_{i, j=1}^{N} \varrho_{i j} \sigma_{i} \sigma_{j}\right)= & \left(\frac{\beta}{2 \pi}\right)^{\frac{N}{2}}(\operatorname{det} \varrho)^{-\frac{1}{2}} \int_{-\infty}^{\infty} \int_{i=1}^{N} \prod_{i=1}^{N} d X_{i} \\
& \cdot \exp \left(-\frac{\beta}{2} \sum_{i, j=1}^{N}\left(\varrho^{-1}\right)_{i j} X_{i} X_{j}+\beta \sum_{i=1}^{N} X_{i} \sigma_{i}\right)
\end{aligned}
$$

which is valid for an arbitrary set $\left\{\sigma_{i}\right\}_{i=1}^{N}$ of commuting operators and any positive definite symmetric matrix $\varrho$. Representing each of the $3 n$ ordered exponentials in (4.5) as multiple integrals according to (4.6) yields

$$
\begin{aligned}
Z_{N, n}(\gamma)= & \left(\frac{\beta}{2 \pi n}\right)^{\frac{3 N n}{2}}(\operatorname{det} J)^{\frac{N n}{2}}(\operatorname{det} \varrho)^{-\frac{3 n}{2}} \int_{-\infty}^{\infty} \int_{i=1}^{N} \prod_{t=1}^{N} d \boldsymbol{X}_{i t} \\
& \cdot \exp \left(-\frac{\beta}{2 n} \sum_{i, j=1}^{N} \sum_{t=1}^{n}\left(\varrho^{-1}\right)_{i j} \boldsymbol{X}_{i t} \cdot \boldsymbol{J} \cdot \boldsymbol{X}_{j t}\right) \\
& \operatorname{re} \operatorname{Tr}\left\{\prod_{t=1}^{n} \prod_{\alpha=x, y, z} \exp \left[\frac{\beta}{n} \sum_{i=1}^{N}\left(\boldsymbol{J} \cdot \boldsymbol{X}_{i t}+\boldsymbol{H}\right)^{\alpha} \sigma_{i}^{\alpha}\right]\right\}
\end{aligned}
$$

where the trace has been taken inside the integrals. 
To proceed any further we need a suitable upper bound on the trace appearing in (4.7). This is supplied by the following:

Lemma. In the notation we have developed

$$
\left|\operatorname{Tr} \prod_{t=1}^{n}\left(e^{x_{t} S^{x}} e^{y_{t} S^{y}} e^{z_{t} S^{z}}\right)\right| \leqq \exp \left\{3 s \sum_{t=1}^{n}\left\|\boldsymbol{x}_{t}\right\|^{2}+\frac{1}{n} \sum_{t=1}^{n} \log C_{s}\left(n s\left\|\boldsymbol{x}_{t}\right\|\right)\right\}
$$

where $\boldsymbol{x}_{\boldsymbol{t}}=\left(x_{t}, y_{t}, z_{t}\right)$.

The proof of the lemma is given in the Appendix.

Factoring out the direct product over $i$ in (4.7) and resorting to the lemma we have

$$
\begin{aligned}
Z_{N, n}(\gamma) \leqq & \left(\frac{\beta}{2 \pi n}\right)^{\frac{3 N n}{2}}(\operatorname{det} J)^{\frac{N n}{2}}(\operatorname{det} \varrho)^{-\frac{3 n}{2}} \int_{-\infty}^{\infty} \int_{i=1}^{N} \prod_{i=1}^{N} \prod_{t=1}^{n} d \boldsymbol{X}_{i t} \\
& \cdot \exp \left\{-\frac{\beta}{2 n} \sum_{i, j=1}^{N} \sum_{t, s=1}^{n}\left[\left(\varrho^{-1}\right)_{i j} \delta_{t s}-\frac{\delta_{i j}}{n z}\right] \boldsymbol{X}_{i t} \cdot \boldsymbol{J} \cdot \boldsymbol{X}_{j s}\right\} \\
& \prod_{i=1}^{N} \exp \left\{-\frac{\beta}{2 n^{2} z} \sum_{t, s=1}^{n} \boldsymbol{X}_{i t} \cdot \boldsymbol{J} \cdot \boldsymbol{X}_{i s}+\frac{1}{n} \sum_{t=1}^{n} \log C_{s}\left(\beta\left\|\boldsymbol{J} \cdot \boldsymbol{X}_{i t}+\boldsymbol{H}\right\|\right)\right. \\
& \left.+\frac{3 \beta^{2}}{n^{2} s} \sum_{t=1}^{n}\left\|\boldsymbol{J} \cdot \boldsymbol{X}_{i t}+\boldsymbol{H}\right\|^{2}\right\}
\end{aligned}
$$

where in anticipation of the next step a term has been added and subtracted in the exponent.

To obtain an upper bound for $Z_{N, n}(\gamma)$ our strategy now is to replace each term in the product over $i$ in (4.9) by the common maximum. This maximum occurs for $\boldsymbol{X}_{i t}=\boldsymbol{X}_{t}$ satisfying the stationary conditions

$$
\frac{1}{n z} \sum_{t=1}^{n} \boldsymbol{X}_{t}=B_{s}\left(\beta\left\|\boldsymbol{J} \cdot \boldsymbol{X}_{t}+\boldsymbol{H}\right\|\right) \frac{\boldsymbol{J} \cdot \boldsymbol{X}_{t}+\boldsymbol{H}}{\left\|\boldsymbol{J} \cdot \boldsymbol{X}_{t}+\boldsymbol{H}\right\|}+\frac{6 \beta}{n s}\left(\boldsymbol{J} \cdot \boldsymbol{X}_{t}+\boldsymbol{H}\right) .
$$

Taking the norm of the relationship (4.10) and inverting it produces $\left\|\boldsymbol{J} \cdot \boldsymbol{X}_{t}+\boldsymbol{H}\right\|$ as a function of $\frac{1}{n z}\left\|\sum_{t=1}^{n} \boldsymbol{X}_{t}\right\|$ which is independent of $t$. Hence we can conclude from (4.10) that the required maximum occurs for $\boldsymbol{X}_{i t}=\boldsymbol{X}$ a solution of

$$
z^{-1} \boldsymbol{X}=B_{s}(\beta\|\boldsymbol{J} \cdot \boldsymbol{X}+\boldsymbol{H}\|) \frac{\boldsymbol{J} \cdot \boldsymbol{X}+\boldsymbol{H}}{\|\boldsymbol{J} \cdot \boldsymbol{X}+\boldsymbol{H}\|}+\frac{6 \beta}{n s}(\boldsymbol{J} \cdot \boldsymbol{X}+\boldsymbol{H}) .
$$

To perform the remaining integral in (4.9) we notice [19] that the eigenvalues of $\left[\left(\varrho^{-1}\right)_{i j} \delta_{t s}-\frac{\delta_{i j}}{n z}\right]$ are given by the $N n$ combinations $\left(\varrho_{i}^{-1}-\frac{\lambda_{t}}{n z}\right)$ where $\varrho_{i}$ are the eigenvalues of $\varrho$ and $\lambda_{1}=n, \lambda_{2}=\lambda_{3} \cdots=\lambda_{n}=0$ are the eigenvalues of an $n \times n$ matrix with entries all unity. The integrations can now be carried out immediately 
after the appropriate orthogonal transformations of variables to give

$$
\begin{aligned}
Z_{N, n}(\gamma) \leqq & {\left[\operatorname{det}\left(I-\frac{\varrho}{z}\right)\right]^{-\frac{3}{2}} } \\
& \cdot \exp \left\{N\left[-\frac{\beta}{2 z} \boldsymbol{X} \cdot \boldsymbol{J} \cdot \boldsymbol{X}+\log C_{s}(\beta\|\boldsymbol{J} \cdot \boldsymbol{X}+\boldsymbol{H}\|)+\frac{3 \beta^{2}}{n s}\|\boldsymbol{J} \cdot \boldsymbol{X}+\boldsymbol{H}\|\right]\right\} .
\end{aligned}
$$

The manipulation leading to (4.12) obviously requires the matrix $I-\frac{\varrho}{z}$ to be positive definite. As is shown in [4], for spins located on the vertices of a regular cubic lattice, this is true provided

$$
z>\gamma^{\nu} \sum_{\boldsymbol{l}} \varrho(\gamma\|\boldsymbol{l}\|)=g_{N}(0, \gamma)
$$

that is, $z$ is greater than the maximum eigenvalue of $\varrho$.

Taking the limit $n \rightarrow \infty$ in (4.12) we have from (4.4) that

$$
Z_{N}(\gamma) \leqq\left[\operatorname{det}\left(I-\frac{\varrho}{z}\right)\right]^{-\frac{3}{2}} \exp \left\{N\left[-\frac{1}{2} \beta z \boldsymbol{x} \cdot \boldsymbol{J} \cdot \boldsymbol{x}+\log C_{s}(\beta\|z \boldsymbol{J} \cdot \boldsymbol{x}+\boldsymbol{H}\|)\right]\right\}
$$

where $\boldsymbol{x}=z^{-1} \boldsymbol{X}$ is the maximizing solution of

$$
\boldsymbol{x}=B_{s}(\beta\|z \boldsymbol{J} \cdot \boldsymbol{x}+\boldsymbol{H}\|) \frac{z \boldsymbol{J} \cdot \boldsymbol{x}+\boldsymbol{H}}{\|z \boldsymbol{J} \cdot \boldsymbol{x}+\boldsymbol{H}\|} .
$$

It now follows from (4.14), (4.13) and (3.2) that

$$
\begin{aligned}
\psi(\gamma)= & -\lim _{N \rightarrow \infty}(\beta N)^{-1} \log Z_{N}(\gamma) \\
\geqq & \frac{1}{2} z \boldsymbol{x} \cdot \boldsymbol{J} \cdot \boldsymbol{x}-\beta^{-1} \log C_{s}(\beta\|z \boldsymbol{J} \cdot \boldsymbol{x}+\boldsymbol{H}\|) \\
& +\lim _{N \rightarrow \infty} \frac{3}{2}(\beta N)^{-1} \log \operatorname{det}\left(I-\frac{\varrho}{z}\right)
\end{aligned}
$$

for all $z>g(0, \gamma)$. Hence taking the limit $z \rightarrow g(0, \gamma)+$ in (4.15) and (4.16), followed by the limit $\gamma \rightarrow 0+, \boldsymbol{x}$ becomes a solution of (2.14) which minimizes the first two terms in (4.16) and consequently

$$
\begin{aligned}
\psi_{0} \geqq & \min _{\boldsymbol{x}}\left\{\frac{1}{2} g(0) \boldsymbol{x} \cdot \boldsymbol{J} \cdot \boldsymbol{x}-\beta^{-1} \log C_{\mathbf{s}}(\beta\|g(0) \boldsymbol{J} \cdot \boldsymbol{x}+\boldsymbol{H}\|)\right\} \\
& +\lim _{\gamma \rightarrow 0+} \lim _{N \rightarrow \infty} \frac{3}{2}(\beta N)^{-1} \log \operatorname{det}\left(I-\frac{\varrho}{g(0, \gamma)}\right) .
\end{aligned}
$$

In view of the upper bound (3.19), the theorem will be proved once we have shown that the last term in (4.17) is zero. For the proof of this fact we refer the reader to [4]. 


\section{Appendix}

Our aim here is to prove the result (4.8)

$$
\left|\operatorname{Tr} \prod_{t=1}^{n}\left(e^{x_{t} S^{x}} e^{y_{t} S^{y}} e^{z_{t} S^{z}}\right)\right| \leqq \exp \left\{3 s \sum_{t=1}^{n}\left\|\boldsymbol{x}_{t}\right\|^{2}+\frac{1}{n} \sum_{t=1}^{n} \log C_{s}\left(n s\left\|\boldsymbol{x}_{t}\right\|\right)\right\}
$$

To obtain the upper bound (A.1) we use the Hölder inequality [20]

with

$$
\left|\operatorname{Tr} \prod_{t=1}^{n} A_{t}\right| \leqq \prod_{t=1}^{n}\left[\operatorname{Tr}\left(A_{t}^{\dagger} A_{t}\right)^{\frac{n}{2}}\right] \frac{1}{n}
$$

$$
A_{t}=e^{x_{t} S^{x}} e^{y_{t} S^{y}} e^{z_{t} S^{z}} .
$$

We now specialize to the spin $-\frac{1}{2}$ case for which we have the formulae

$$
\begin{gathered}
e^{\boldsymbol{x} \cdot \boldsymbol{S}}=\cosh \left(\frac{1}{2}\|x\|\right)+\hat{x} \cdot \boldsymbol{S} \sinh \left(\frac{1}{2}\|x\|\right), \\
(\boldsymbol{x} \cdot \boldsymbol{S})(\boldsymbol{y} \cdot \boldsymbol{S})=\boldsymbol{x} \cdot \boldsymbol{y}+i \boldsymbol{S} \cdot(\boldsymbol{x} \times \boldsymbol{y}) .
\end{gathered}
$$

The general spin case follows straightforwardly from the spin $-\frac{1}{2}$ case. Using (A.4) and (A.5) and abbreviating the hyperbolic functions

$$
c_{\alpha}=\cosh \alpha_{t}, \quad t_{\alpha}=\tanh \alpha_{t} \quad(\alpha=x, y, z)
$$

we reach the quaternionic form for (A.3)

where

$$
A_{t}=a_{0}(1+\boldsymbol{a} \cdot \boldsymbol{S})
$$

and

$$
a_{o}=c_{x} c_{y} c_{z}\left(1+i t_{x} t_{y} t_{z}\right)
$$

$$
\boldsymbol{a}=\left(t_{x}+i t_{y} t_{z}, t_{y}+i t_{z} t_{x}, t_{z}+i t_{x} t_{y}\right) .
$$

We can now calculate

where

$$
\begin{aligned}
A_{t}^{\dagger} A_{t} & =\left|a_{0}\right|^{2}\left[1+\|\boldsymbol{a}\|^{2}+\left(\boldsymbol{a}+\boldsymbol{a}^{*}+i\left(\boldsymbol{a}^{*} \times \boldsymbol{a}\right)\right) \cdot \boldsymbol{S}\right] \\
& =\left|a_{0}\right|^{2}\left(1+\|\boldsymbol{a}\|^{2}\right)(1+\boldsymbol{b} \cdot \boldsymbol{S}) \\
\boldsymbol{b} & =2\left(1+\|\boldsymbol{a}\|^{2}\right)^{-1}(\operatorname{re} \boldsymbol{a}-\operatorname{re} \boldsymbol{a} \times \operatorname{im} \boldsymbol{a}) .
\end{aligned}
$$

Since $\boldsymbol{b}$ is real the eigenvalues of $\boldsymbol{b} \cdot \boldsymbol{S}$ are $\frac{1}{2}\|\boldsymbol{b}\|$ and $-\frac{1}{2}\|\boldsymbol{b}\|$, hence from (A.10) we deduce that

$$
\operatorname{Tr}\left(A_{t}^{\dagger} A_{t}\right)^{\frac{n}{2}}=\left[\left|a_{0}\right|^{2}\left(1+\|\boldsymbol{a}\|^{2}\right)\right]^{\frac{n}{2}}\left\{\left(1+\frac{1}{2}\|\boldsymbol{b}\|\right)^{\frac{n}{2}}+\left(1-\frac{1}{2}\|\boldsymbol{b}\|\right)^{\frac{n}{2}}\right\} .
$$

Furthermore the inequality

implies

$$
1+x \leqq e^{x}
$$

$$
\left(1+\frac{1}{2}\|\boldsymbol{b}\|\right)^{\frac{n}{2}}+\left(1-\frac{1}{2}\|\boldsymbol{b}\|\right)^{\frac{n}{2}} \leqq 2 \cosh \frac{n\|\boldsymbol{b}\|}{4}
$$

and in conjunction with the inequalities

$$
\cosh x \leqq e^{\frac{1}{2} x^{2}}, \quad \tanh ^{2} x \leqq x^{2}
$$


leads to

$$
\begin{aligned}
\left|a_{0}\right|^{2}\left(1+\|\boldsymbol{a}\|^{2}\right) & =c_{x}^{2} c_{y}^{2} c_{z}^{2}\left(1+t_{x}^{2} t_{y}^{2} t_{z}^{2}\right)\left(1+t_{x}^{2}+t_{y}^{2}+t_{z}^{2}+t_{x}^{2} t_{y}^{2}+t_{y}^{2} t_{z}^{2}+t_{z}^{2} t_{x}^{2}\right) \\
& \leqq e^{\left\|\boldsymbol{x}_{t}\right\|^{2}}\left\{\left(1+t_{x}^{2}\right)\left(1+t_{y}^{2}\right)\left(1+t_{z}^{2}\right)\right\}^{2} \\
& \leqq e^{\left\|\boldsymbol{x}_{t}^{2}\right\|} e^{2\left(t_{x}^{2}+t_{y}^{2}+t_{z}^{2}\right)} \leqq e^{3\left\|\boldsymbol{x}_{t}\right\|^{2}}
\end{aligned}
$$

Finally using (A.11) we have

$$
\begin{aligned}
\left(\frac{1}{2}\|\boldsymbol{b}\|\right)^{2} & =\left(1+\|\boldsymbol{a}\|^{2}\right)^{-2}\left(\| \text { rea } \boldsymbol{a}\left\|^{2}+\right\| \text { re } \boldsymbol{a} \times \operatorname{im} \boldsymbol{a} \|^{2}\right) \\
& \leqq\left(1+\|\boldsymbol{a}\|^{2}\right)^{-2}\left(1+\|\operatorname{im} \boldsymbol{a}\|^{2}\right) \cdot \| \text { re } \boldsymbol{a} \|^{2} \\
& \leqq \| \text { re } \boldsymbol{a} \|^{2}=t_{x}^{2}+t_{y}^{2}+t_{z}^{2} \\
& \leqq\left\|\boldsymbol{x}_{t}\right\|^{2} .
\end{aligned}
$$

Applying the inequalities (A.14), (A.16) and (A.17) collectively to (A.12) yields

$$
\operatorname{Tr}\left(A_{t}^{\dagger} A_{t}\right)^{\frac{n}{2}} \leqq e^{\frac{3 n}{2}\left\|\boldsymbol{x}_{t}\right\|^{2}} \cdot 2 \cosh \left(\frac{n}{2}\left\|\boldsymbol{x}_{t}\right\|\right)
$$

which when substituted in (A. 2) gives the required result (A.1) for $s=\frac{1}{2}$.

The extension to general spin is achieved through the

Proposition. For any real vectors $\boldsymbol{x}$ and $\boldsymbol{y}$ there is a polar decomposition

$$
e^{\boldsymbol{x} \cdot \boldsymbol{S}} e^{\boldsymbol{y} \cdot \boldsymbol{S}}=e^{\boldsymbol{\alpha} \cdot \boldsymbol{s}} e^{i \boldsymbol{\beta} \cdot \boldsymbol{S}}
$$

where the real vectors $\boldsymbol{\alpha}, \boldsymbol{\beta}(\bmod 4 \pi \hat{\boldsymbol{y}})$ are unique and independent of the spin.

We postpone the proof of the proposition but notice that by the closure of the rotation group (i.e. operators of the form $e^{i \boldsymbol{\beta} \cdot \boldsymbol{s}}$ ) under composition it has as a consequence the fact that we can write uniquely

$$
\left(A_{t}^{\dagger} A_{t}\right)^{\frac{1}{2}}=e^{\boldsymbol{\alpha}_{t} \cdot \boldsymbol{S}}
$$

where the real vectors $\alpha_{t}$ are appropriate for all values of the spin. The spin $-\frac{1}{2}$ inequality (A.18) now reads, for any integer $n$

$$
\operatorname{Tr} e^{n \boldsymbol{\alpha}_{t} \cdot \boldsymbol{S}}=2 \cosh \left(\frac{n}{2}\left\|\boldsymbol{\alpha}_{t}\right\|\right) \leqq e^{\frac{3 n}{2}\left\|\boldsymbol{x}_{t}\right\|^{2}} \cdot 2 \cosh \left(\frac{n}{2}\left\|\boldsymbol{x}_{t}\right\|\right)
$$

Hence for general spin we have

$$
\begin{aligned}
\operatorname{Tr}\left(A_{t}^{\dagger} A_{t}\right)^{\frac{n}{2}} & =\operatorname{Tr} e^{n \boldsymbol{\alpha}_{t} \cdot \boldsymbol{s}}=C_{s}\left(n s\left\|\boldsymbol{\alpha}_{t}\right\|\right) \\
& = \begin{cases}1+\sum_{m=1}^{s} 2 \cosh \left(m n\left\|\boldsymbol{\alpha}_{t}\right\|\right) & s \text { integral } \\
\sum_{m=\frac{1}{2}}^{s} 2 \cosh \left(m n\left\|\boldsymbol{\alpha}_{t}\right\|\right) & s \text { half integral }\end{cases} \\
& \leqq e^{3 n s\left\|\boldsymbol{x}_{t}\right\|^{2}} C_{s}\left(n s\left\|\boldsymbol{x}_{t}\right\|\right)
\end{aligned}
$$


In view of (A.2) this establishes the result (A.1). We now return to the

Proof of the Proposition. The coefficient vectors $\boldsymbol{\alpha}, \boldsymbol{\beta}$ can in principle be determined from the relations

$$
\begin{aligned}
& e^{2 \boldsymbol{\alpha} \cdot \boldsymbol{S}}=\left(e^{\boldsymbol{x} \cdot \boldsymbol{S}} e^{\boldsymbol{y} \cdot \boldsymbol{S}}\right)^{\dagger}\left(e^{\boldsymbol{x} \cdot \boldsymbol{S}} e^{\boldsymbol{y} \cdot \boldsymbol{S}}\right) \\
& e^{i \boldsymbol{\beta} \cdot \boldsymbol{S}}=e^{-\boldsymbol{\alpha} \cdot \boldsymbol{S}} e^{\boldsymbol{x} \cdot \boldsymbol{s}} e^{\boldsymbol{y} \cdot \boldsymbol{S}}
\end{aligned}
$$

using the Baker-Campbell-Hausdorff formula [21]

$$
e^{\boldsymbol{x} \cdot \boldsymbol{S}} e^{\boldsymbol{y} \cdot \boldsymbol{S}}=e^{\boldsymbol{x} \cdot \boldsymbol{S}+\boldsymbol{y} \cdot \boldsymbol{S}+\frac{1}{2}[\boldsymbol{x} \cdot \boldsymbol{S}, \boldsymbol{y} \cdot \boldsymbol{S}]+\frac{1}{12}[[\boldsymbol{x} \cdot \boldsymbol{S}, \boldsymbol{y} \cdot \boldsymbol{S}], \boldsymbol{y} \cdot \boldsymbol{S}]+\cdots}
$$

which involves only manipulations within the Lie algebra. Since the Lie algebras for different spins are isomorphic (over the complex field) the desired result follows from the existence and 'uniqueness' of the representation (A.19) for $\operatorname{spin}-\frac{1}{2}$.

Acknowledgement. We would like to thank Drs. Tindemans and Capel for sending preprints of their work prior to publication. Paul A. Pearce acknowledges support from a Commonwealth post-graduate award.

\section{References}

1. Lebowitz, J.L., Penrose, O.: J. Math. Phys. 7, 98 (1966)

2. Lieb,E.H.: J. Math. Phys. 7, 1016 (1966)

3. Thompson, C.J.: Mathematical statistical mechanics, Appendix C. New York: Macmillan 1972

4. Thompson, C. J., Silver, H.: Commun. math. Phys. 33, 53 (1973)

5. Silver, H., Frankel, N.E., Ninham, B. W.: J. Math. Phys. 13, 468 (1972)

6. Millard, K., Leff, H.: J. Math. Phys. 12, 1000 (1971)

7. Lieb, E.H.: Commun. math. Phys. 31, 327 (1973)

8. Griffiths, R. B.: Phase transitions and critical phenomena. Eds. Domb, C., Green, M.S., London: Academic Press 1972

9. Van Vleck, J.H.: Theory of electric and magnetic susceptibilities, p. 316. Oxford: Oxford University Press 1932

10. Brout, R.: Phase transitions, p. 30. New York: Benjamin 1965

11. Kittel, C., Shore, H.: Phys. Rev. 138, A 1165 (1965)

12. Niemeyer, Th.: Physica 48, 467 (1970)

13. Vertogen, G., De Vries, A. S. : Physica 59, 634 (1972)

14. Trotter,H.F.: Pacific J. Math. 8, 887 (1958)

15. Tindemans, P.A. J., Capel, H. W.: Physica 72, 433 (1974)

16. Tindemans, P. A. J., Capel, H. W.: Physica 75, 407 (1974)

17. Girardeau, M.: J. Math. Phys. 3, 131 (1962)

18 Huber, A.: Methods and problems of theoretical physics. Ed. Bowcock, J. E., Amsterdam: NorthHolland 1970

19. Lancaster, P.: Theory of matrices, p. 259. New York: Academic Press 1969

20. Mehta, C.L.: J Math. Phys. 9, 693 (1968)

21. Weiss, G.H., Maradudin, A. A. : J. Math. Phys. 3, 760 (1962)

Communicated by G. Gallavotti

Paul A. Pearce

Mathematics Department

University of Melbourne

Parkville, Victoria, Australia 
